\title{
BUZANO'S INEQUALITY IN ALGEBRAIC PROBABILITY SPACES
}

\author{
J. Agredo, Y. Leon, J. Osorio And A. PeÑA
}

Abstract. We obtain a generalization of Buzano's inequality of vectors in Hilbert spaces , using the theory of algebraic probability spaces. In particular, we extend a result of Dragomir given in [?]. Applications for numerical inequalities for $n$-tuples of bounded linear operators and functions of operators defined by double power series are also generalized.

Mathematics subject classification (2010): 47A05, 47A63, 46L30.

Keywords and phrases: Buzano's inequality, algebraic probability spaces.

\section{REFERENCES}

[1] J. Agredo, F. Fagnola, R. Rebolledo, Decoherence free subspaces of a quantum Markov semigroup, J. Math. Phys., 55, 112201 (2014).

[2] S. ATtal, Elements of Operators Algebras and Modular Theory, Open Quantum Systems I: The Hamiltonian approach, Springer Verlag, Lectures Notes in Mathematics, 2006, 1-105.

[3] O. Bratelli, D. W. Robinson, Operator Algebras and Quantum Statistical Mechanics, SpringerVerlag 1, 1987.

[4] M. L. Buzano, Generalizzazione della diseguaglianza di Cauchy-Schwartz, Rend. Sem. Mat. Univ. e Politech. Torino, 31, (1974), 405-409.

[5] A. Connes, Noncommutative Geometry, Academic Press, 1994.

[6] F. D'AndREA, Pythagoras Theorem in Noncommutative Geometry, Proceedings of the Conference on Optimal Transport and Noncommutative Geometry, Besancon 2014, Available at arXiv:1507.08773.

[7] S. S. Dragomir, Generalizations of Buzano inequality for $n$-tuples of vectors in inner product spaces with applications, Tbilisi Mathematical Journal, 10, 2 (2017), 29-41.

[8] S. S. Dragomir, M. Khosravi, M. S. Moslehian, Bessel type inequalities in Hilbert modules, Linear Multilinear Algebra, 58, 8 (2010), 967-975.

[9] S. S. DRAGomir, Some inequalities for the norm and the numerical radius of linear operators in Hilbert spaces, Tamkang J. Math., 39, 1 (2008), 1-7.

[10] S. S. DRAGOMIR, Inequalities for the norm and the numerical radius of linear operators in Hilbert spaces, Demostratio Math., 40, 2 (2007), 411-417.

[11] S. S. DRAGOMIR, A potpourri of Schwarz related inequalities in inner product spaces (II), J. Inequal. Pure Appl. Math.; 7, 1 (2006), Article 14.

[12] S. S. DRAGOMIR, I. SÁNDOR, Some inequalities in pre-Hilbertian spaces, Studia Univ. Babes-Bolyai Math., 32, 1 (1987), 71-78.

[13] S. S. DRAGOMIR, Some refinements of Schwartz inequality, Simpozionul de Matematici si Aplicatii, Timisoaria, Romania, 1-2 Noiembrie, (1985), 13-16.

[14] F. Fagnola, V. Umanit 'A, Generic quantum Markov semigroups, cycle decomposition and deviation from equilibrium, Infin. Dimens. Anal. Quantum Probab. Relat. Top., 15, 3 (2012), 1-17.

[15] F. FAGNOLA, R. REBOLlEDO, Algebraic conditions for convergence of a quantum Markov semigroup to a steady state, Infin. Dimens. Anal. Quantum Probab. Relat. Top., 11, 3 (2008), 467-474.

[16] F. Fagnola, R. Rebolledo, Subharmonic projections for a quantum Markov semigroup, J. Math. Phys., 43, 2 (2002).

[17] F. Fagnola, Quantum Markov semigroups and quantum Markov flows, Proyecciones., 18, 3 (1999), $1-144$. 
[18] J. I. FujiI, M. FujiI, M. S. Moslehian, J. E. Pecaric, Y. Seo, Reverses Cauchy-Schwarz type inequalities in pre-inner product $C^{*}$-modules, Hokkaido Math. J., 40, (2011), 1-17.

[19] M. FUJII, Operator-valued inner product and operator inequalities, Banach J. Math. Anal., 2, 2 (2008), 59-67.

[20] M. FujII, F. Kubo, Buzano's inequality and bounds for roots of algebraic equations, Proc. Amer. Math. Soc., 117, (1993), 359-361.

[21] D. Ilisevic, S. VARos ANeC, On the Cauchy-Schwarz inequality and its reverse in semi-inner product $C^{*}$-modules, Banach J. Math. Anal., 1, (2007), 78-84.

[22] M. S. Moslehian, L. - E. Persson, Reverse Cauchy-Schwarz inequalities for positive $C^{*}$-valued sesquilinear forms, Math. Inequal. Appl., 4, 12, (2009), 701-709.

[23] A. Hora, N. Obata, Quantum Probability and Spectral Analysis of Graphs, Series: Theoretical and Mathematical Physics, Springer, Berlin Heidelberg, 2007.

[24] K. R. Parthasarathy, An Introduction to Quantum Stochastic Calculus, Monographs in Mathematics 85, 1992.

[25] U. RICHARD, Sur des inégalités du type Wirtinger et leurs application aux équationes différentielles ordinaires, Colloquium of Analysis held in Rio de Janeiro, (1972), 233-244.

[26] W. Rudin, Real and complex analysis, McGraw-Hill, 1987.

[27] S. SALIMI, Continuous-time quantum walks on semi-regular spidernet graphs via quantum probability theory, Quantum Information Processing, 9, 1 (2010), 75-91.

[28] S. S ALImi, Quantum Central Limit Theorem for Continuous-Time Quantum Walks on Odd Graphs in Quantum Probability Theory, International Journal of Theoretical Physics, 47, 12, (2008), 3298-3309.

[29] S. SALIMI, Study of continuous-time quantum walks on quotient graphs via quantum probability theory, International Journal of Quantum Information, 6, 4, (2008), 945-957.

[30] K. Tanahashi, A. Uchiyama, M. Uchiyama, On Schwarz type inequalities, Proc. Amer. Math. Soc., 131, 8, (2003) 2549-2552.

[31] M. TAKESAKI, Theory of operator algebras I, Springer-Verlag New York Heidelberg, 1979. 